\title{
False Positive Diagnosis of Lysosomal Storage Disease Based on Dried Blood Spot Sample; Leucocyte Number of a Challenging Factor
}

\author{
(D) Eser Yıldırım Sözmen1, (1) Meral Dondurmacı1, (1) Sema Kalkan Uçar2, (1) Mahmut Çoker2 \\ ${ }^{1}$ Ege University Faculty of Medicine, Department of Medical Biochemistry and Metabolism Laboratory, Izmir, Turkey \\ 2Ege University Faculty of Medicine, Department of Pediatric Metabolic Disease, Izmir, Turkey
}

\begin{abstract}
Aim: Recently dried blood spot (DBS) samples have been recommended as a screening test for Lysosomal Storage diseases. Although DBS samples have many advantages including non-invasiveness, cost and transportation, usage of these samples is limited by its high false positive rate. We aimed to investigate any possible effect of the leucocyte number on enzyme activity in dried blood samples in a retrospective study.

Materials and Methods: Data was collected from subjects $(n=263)$ for whom hematological parameters were available in the database of Ege University Hospital. The lysosomal enzyme activity results (alpha glycosidase, glycocerebrosidase, alpha galactosidase, sphingomyelinase and galactocerebrosidase) were re-evaluated with regard to the leucocyte number. Enzyme activities were measured using fluorometric and liquid chromatography-tandem mass spectrometry methods.

Results: All enzyme activities closely correlated with the total number of leucocyte, since leucocytes are the main source of lysosomal enzymes. Glycocerebrosidase and galactocerebrosidase presented a positive correlation with the number of neutrophils and sphingomyelinase showed a positive correlation with the number of lymphocytes. When we recalculated the lysosomal enzyme activities with regard to the leucocyte number, the false positive rates for glycocerebrosidase, sphingomyelinase and alpha galactosidase decreased from $20 \%, 10.5 \%$ and $10.8 \%$ to $4.5 \%, 4.4 \%$ and $4.2 \%$, respectively.

Conclusions: Our data indicated that the enzyme activity in dried blood samples including low leucocyte number might be found lower than reference intervals resulting in false positive diagnosis. We concluded that the calculation of enzyme activity with regard to the number of leucocytes might produce more reliable results and might be helpful in decreasing the false positive rate.

Keywords: Dried blood spot, leucocyte number, lysosomal storage disease
\end{abstract}

\section{Introduction}

Lysosomal Storage diseases (LSD), which are related to a deficiency of specific lysosomal hydrolases, resulted in clinical aspects due to an accumulation of substrates in different tissues. It is critically important to diagnose patients in the early stages of this disease since enzyme replacement therapy might prevent complications and also increase life expectancy. Although the determination of enzyme activities in leucocyte and/or fibroblast samples are recommended as the gold standard, these samples are not preferred for screening due to their disadvantages; namely; sampling is invasive, the method is expensive, fast transportation is essential etc (1-3). Since dried blood spot (DBS) is non-invasive, low-cost, easily transportable, has acceptable enzyme stability compared to leucocyte and/or 
fibroblast culture, it is recommended as a first screening test. However the false positive rate with DBS sample is higher compared to other samples (1-3). DBS is a sample from the total blood of subjects, it contains erythrocytes, leucocytes, thrombocytes which might affect the enzyme activity measurement. Raghavan et al. (4) firstly pointed out that the total specific activity of a Gaucher heterozygote might lead to misdiagnosis due to the variation in enzyme activities in different types of leucocyte. Since then, many studies have been conducted to produce greater test accuracy and to increase the diagnostic efficacy of DBS samples (5-7). The strict control of storage conditions (such as humidity, temperature and so on), the correct preparation of the sample, sample blank correction and participation in quality control programs are recommended by many authors in order to validate these methods. Recently, the specificity and sensitivity of DBS enzyme assays to diagnose LSD have been reported as 95.2\% (92-96\%) and $85 \%(64-100 \%)$ respectively $(5,6)$. Even though all above mentioned factors were controlled, the false positive rates are still high because of individual factors, such as pseudo deficiency due to isozymes and so on. The determination of enzyme activities in leucocyte samples were accepted as the gold standard test because of the corrected calculation of enzyme activity regarding protein levels in leucocytes and its high sensitivity and specificity. Although some authors investigated the factors which affect lysosomal enzyme activity in leucocyte and the enzyme activities in different types of leucocytes $(4,8-10)$, there is no data on the effect of hemogram parameters, especially leucocyte numbers, on the enzyme activities in DBS samples. We investigated any possible effects of these parameters on enzyme activity measurements in dried blood samples in a retrospective study in order to explain the false positive rate due to individual factors.

\section{Materials and Methods}

This study was approved by the Local Ethics Committee of Ege University (approval number: 16-8/2). Data was collected from the subjects for whom hematological parameters (hemoglobin, hematocrit, leucocyte, neutrophil, lymphocyte and thrombocyte) were available in the database of Ege University Hospital. Totally 1500 subjects clinically suspected had been screened for LSD from January 2015 to September 2016. Clinically and genetically verified patients were excluded. Two hundred sixty three subjects of this population had hematologic test results. Lysosome enzyme activities had been determined by the ultra-high pressure liquid chromatography-tandem mass spectrometer (MS/ MS) method (sphingomyelinase and galactocerebrosidase activities) and fluorometric method ( $\alpha$-galactosidase, $\alpha$-glycosidase, glycocerebrosidase activities) and hematological parameters had been determined by routine laboratory methods.

\section{Statistical Analysis}

All data were evaluated using the SPSS 22.0 statistics program and comparisons were made using the Student t-test, correlations were calculated using the Pearson test.

\section{Results}

The general characteristics of population are presented in Table I. Glycocerebrosidase enzyme activity showed a positive correlation with the total number of leukocytes and the number of neutrophils (Figure 1a). Sphingomyelinase enzyme activity showed a positive correlation with the total number of leukocytes and the number of lymphocytes (Figure 1b). Alpha galactosidase enzyme activity showed a positive correlation with the total number of leukocytes and the number of lymphocytes as well as the number of neutrophils (Figure 1c). Interestingly, alpha galactosidase activity correlated with the number of thrombocytes $(r=0.373, p=0.000)$. Alpha glycosidase activity positively correlated with the total number of leucocytes, the number of neutrophils and the number of lymphocytes and the number of thrombocytes $(r=0.197, p=0.007)$ (Figure 1d). Galactocerebrosidase activity positively correlated with the total number of leucocytes, the number of neutrophils and the number of thrombocytes $(r=0.142, p=0.001)$ (Figure $1 \mathrm{e})$. There was no correlation between enzyme activities and hemoglobin/hematocrit levels. Subjects $(n=53)$ with glycocerebrosidase enzyme activity lower than $0.9 \mathrm{nmol} /$ $\mathrm{mL} / \mathrm{h}$ (which is the cut off value to recall the patients) exhibited a significantly lower number for leukocyte $(p=0.02)$ and lymphocyte $(p=0.015)$ compared to those of subjects with enzyme activity higher than 0.9. Subjects $(n=19)$ with sphingomyelinase enzyme activity lower than $3.7 \mathrm{nmol} / \mathrm{mL} / \mathrm{h}$ (which is the cut off value to recall the patients) exhibited a significantly lower number of lymphocyte $(p=0.015)$ compared to those of subjects with enzyme activity higher than 3.7. Subjects $(n=23)$ with

\begin{tabular}{|l|l|l|}
\hline \multicolumn{3}{|l|}{ Table I. General characteristic of study population } \\
\hline & $\mathbf{n}$ & Mean \pm SD \\
\hline Gender (male/female) & 263 & $128 / 135$ \\
\hline Glycocerebrosidase $(\mathrm{nmol} / \mathrm{mL} / \mathrm{h})$ & 263 & $1.87 \pm 1.44$ \\
\hline$\alpha$ glycosidase $(\mathrm{nmol} / \mathrm{mL} / \mathrm{h})$ & 198 & $2.62 \pm 1.34$ \\
\hline$\alpha$ galactosidase $(\mathrm{nmol} / \mathrm{mL} / \mathrm{h})$ & 213 & $6.03 \pm 3.67$ \\
\hline Sphingomyelinase $(\mathrm{nmol} / \mathrm{mL} / \mathrm{h})$ & 181 & $10.32 \pm 7.81$ \\
\hline Galactocerebrosidase $(\mathrm{nmol} / \mathrm{mL} / \mathrm{h})$ & 170 & $1.33 \pm 0.89$ \\
\hline Hemoglobin $(\mathrm{g} / \mathrm{dL})$ & 263 & $11.94 \pm 1.89$ \\
\hline Hematocrite $(\%)$ & 263 & $36.71 \pm 5.43$ \\
\hline Total leucocyte/mm $3 m^{3}$ & 263 & $8.916 \pm 3.748$ \\
\hline Neutrophil number $/ \mathrm{mm}^{3}$ & 263 & $3.861 \pm 2.261$ \\
\hline Lymphocyte number $/ \mathrm{mm}^{3}$ & 263 & $3.846 \pm 2.482$ \\
\hline Thrombocyte number $/ \mathrm{mm}^{3}$ & 263 & $291.839 \pm 122.747$ \\
\hline
\end{tabular}

SD: Standard deviation 
alpha galactosidase enzyme activity lower than $2.2 \mathrm{nmol} /$ $\mathrm{mL} / \mathrm{h}$ (which is the cut off value to recall the patients) exhibited a significantly lower number of thrombocyte $(p=0.015)$ compared to those of subjects with enzyme activity higher than 2.2. Since there was only one subject with low alpha glycosidase activity and 3 subjects with low galactocerebrosidase activity according to their respective cut off limits, they could not be analyzed statistically. We recalculated the estimated leucocyte enzyme activities by dividing the enzyme activities of DBS by the leucocyte number and the reference intervals of estimated leucocyte enzyme activities for healthy population were determined using the nonparametric method according to the procedure recommended by the International Federation of Clinical Chemistry and National Committee for Clinical Laboratory Standards (11). A one-sided reference region $\left(5^{\text {th }}\right.$ percentile) at the lower end was accepted as the decisionmaking limit and data was re-evaluated with regard to this limit of estimated leucocyte enzyme activities. When we recalculated the glycocerebrosidase enzyme activities with regard to the leucocyte number, the false positive number declined from 53 to 12 . While 53 of subjects had had lower enzyme activities than the cut off values, only 12 of these 53 subjects had lower "estimated leucocyte glycocerebrosidase activity" (the glycocerebrosidase/
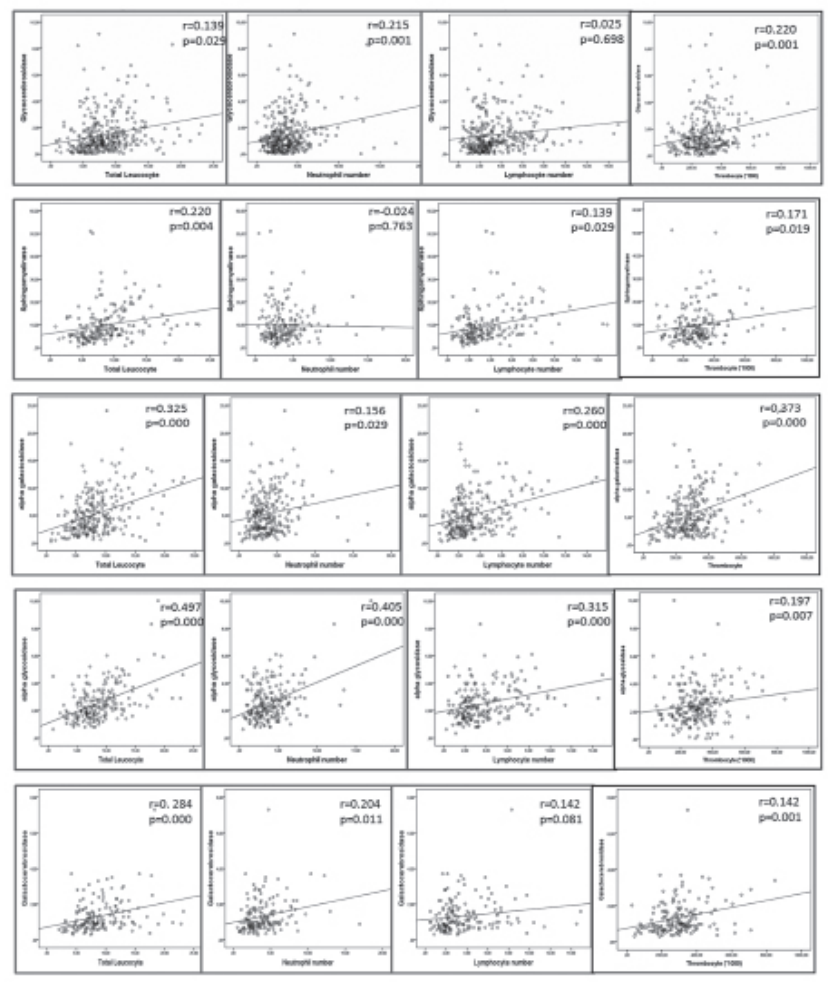

Figure 1. a-e. a) Correlation between the glycocerebrosidase activity and leucocytes, thrombocyte, b) Correlation between the Sphingomyelinase activity and leucocytes, thrombocyte, c) Correlation between alpha galactosidase activity and leucocytes, thrombocyte, d) Correlation between alpha glycosidase activity and leucocytes, thrombocyte, e) Correlation between galactocerebrosidase activity and leucocytes, thrombocyte leucocyte number) after making a correction based on the leucocyte number. Similarly, of the 19 subjects who originally had low sphingomyelinase activity, only 8 still had low estimated leucocyte sphingomyelinase activity, 11 of the 19 subjects exhibited normal estimated leucocyte sphingomyelinase activity. Similarly, of the 23 subjects with low alpha galactosidase activity, only 9 still had low estimated leucocyte alpha galactosidase activity, 14 of 23 subjects exhibited normal estimated leucocyte alpha galactosidase activity. From these data, the false positive rates for glycocerebrosidase, sphingomyelinase and alpha galactosidase decreased via a correction activity based on the leucocyte number from $20 \%, 10.5 \%$ and $10.8 \%$ to $4.5 \%, 4.4 \%$ and $4.2 \%$, respectively.

\section{Discussion}

Although DBS samples are the most suitable material for screening and diagnosis of LSD, interferences related to sample quality might lead to misdiagnosis and/or false positive/negative test results. We investigated the relationship between the hematologic parameters of patients and enzyme activity in DBS materials. Since we corrected the enzyme activity calculation using sample blank to prevent hemoglobin interferences, there was no correlation between the hematocrit/hemoglobin levels and enzyme activity (12). In this study, we showed that all enzyme activities in DBS are closely correlated with the total number of leucocyte, since leucocytes are the main source of lysosomal enzymes. While glycocerebrosidase and galactocerebrosidase presented a positive correlation with the number of neutrophils, sphingomyelinase showed a positive correlation with the number of lymphocytes. Alpha galactosidase and alpha glycosidase enzyme activity were correlated both with neutrophils and lymphocytes. Similarly, Nakagawa et al. (13) found that $\beta$-glucosidase in all cell types is mainly membrane bound and it is stimulated by taurocholate in granulocytes, monocytes and lymphocytes, so they suggested that variations in the total number of leukocytes and in the relative proportion of their various cell types might lead to inconsistent or unreliable values for enzyme activity in the diagnosis of LSD and in carrier detection. Daitx et al. (14) observed no correlation between the enzyme activities in DBS and in leukocytes for the whole population because of the corrected calculation of enzyme activity regarding protein levels in leucocytes and the inhibition of alpha-galactosidase B isozyme activity by $\mathrm{N}$-acetyl-D-galactosamine in DBS samples but not inhibition of isozyme in leucocyte samples (14). Elbin et al. (7) demonstrated that the enzyme activities can be affected by inadequate blood spot processing especially by an incomplete mixing of blood before spotting (7) in accordance with these observations, we showed that the enzyme activities in DBS sample are closely correlated with the number of leucocytes of patients. From this data, we concluded that the calculation of enzyme activity 
with regard to the number of leucocytes might produce more reliable results and might help to decrease the false positive rate. However, it is crucially important to decide the cut off levels of enzymes for diagnosis and for re-testing. Recently, selective screening based on the main clinical findings in LSD has been recommended in the diagnosis of adult patients. Anemia and chronic renal insufficiency are common in Fabry patients and the screening studies among dialysis patients and/or anemia were conducted. Kleinert et al. (15) showed a high prevalence of anemia in 345 adults with Fabry disease from the international database, the Fabry Outcome Survey and proposed kidney failure, heart failure and/or inflammation as potential causes of anemia in Fabry disease (15). Although the main cause of anemia in Fabry disease patients is not clearly understood, it is speculated that the reasons were bone marrow accumulation of globotriaosylceramide or renal insufficiency. However, anemia might be proposed as a parameter to promote selective screening for Fabry disease from this data, the conflicting effect of anemia on enzyme activity in DBS sample should be taken into account for screening studies. Our data indicated that the enzyme activity in dried blood samples including low leucocyte number might be found lower than reference intervals resulting in false positive diagnosis. Therefore, we suggest that the laboratory scientists should evaluate the number of leucocyte while interpreting data. From this data, we concluded that the calculation of enzyme activity with regard to the number of leucocytes might achieve more reliable results and might be helpful in decreasing the false positive rate.

\section{Study Limitations}

Since patients with LSD were excluded from this study, we could not calculate the false-negative rate and also the specificity and sensitivity of estimated leucocyte enzyme activity.

\section{Conclusion}

DBS is the most advantageous matrix for the screening for LSD. However, many factors including hemoglobin levels, transport conditions and/or leukocyte levels might affect the enzyme activities in DBS. Our study showed that a low number of leukocytes is one of the critical factors affecting enzyme activity. Therefore, it is crucially important to interpret the data taking into account the leukocyte number in order to decrease the number of recall patients and the false-positive ratio.

\section{Ethics}

Ethics Committee Approval: This study was approved by Local Ethics Committee of Ege University (approval number: 16-8/2).
Informed Consent: Retrospective study.

Peer-review: External and internal peer-reviewed.

\section{Authorship Contributions}

Surgical and Medical Practices: S.K.U., M.Ç., Concept: E.Y.S., Design: E.Y.S., Data Collection or Processing: M.D., E.Y.S., Analysis or Interpretation: E.Y.S., S.K.U., M.Ç., Literature Search: M.D., E.Y.S., Writing: E.Y.S., S.K.U., M.Ç.

Conflict of Interest: No conflict of interest was declared by the authors.

Financial Disclosure: The authors declared that this study received no financial support.

\section{References}

1. Kingma SDK, Bodamer OA, Wijburg FA. Epidemiology and diagnosis of lysosomal storage disorders; challenges of screening. Best Pract Res Clin Endocrinol Metab 2015;29:145-57.

2. Reusera AJ, Verheijena FW, Balib D, et al. The use of dried blood spot samples in the diagnosis of lysosomal storage disorders-Current status and perspectives. Mol Genet Metab 2011;104:144-8

3. de Jesus VR, Zhang XK, Keutzer $C$, et al. Development and evaluation of quality control dried blood spot materials in newborn screening for lysosomal storage disorders. Clin Chem 2009;55:158-64.

4. Raghavan SS, Topol J, Kolodny EH. Leukocyte ,8-Glucosidase in Homozygotes and Heterozygotes for Gaucher Disease. Am J Hum Genet 1980;32:158-73.

5. Verma J, Thomas DC, Kasper DC, et al. Inherited Metabolic Disorders: Efficacy of Enzyme Assays on Dried Blood Spots for the Diagnosis of Lysosomal Storage Disorders. JIMD Rep 2017;31:15-27.

6. Stroppiano $M$, Calevo MG, Corsolini $F$, et al. Validity of $\beta$-D-glucosidase activity measured in dried blood samples for detection of potential Gaucher disease patients. Clin Biochem 2014;47:1293-6.

7. Elbin CS, Olivova P, Marashio CA, et al. The effect of preparation, storage and shipping of dried blood spots on the activity of five lysosomal enzymes. Clin Chim Acta 2011;412:1207-12.

8. Olivova P, Veen K, Van Der, et al. Effect of sample collection on $\alpha$-galactosidase A enzyme activity measurements in dried blood spots on fi Iter paper. Clin Chim Acta 2009;403:15962.

9. Burin M, Dutra-Filho C, Brum J, et al. Effect of collection, transport, processing and storage of blood specimens on the activity of lysosomal enzymes in plasma and leukocytes. Braz J Med Biol Res 2000;33:1003-13.

10. Lukacs Z, Cobos PN, Mengel E, et al. Diagnostic efficacy of the fluorometric determination of enzyme activity for Pompe disease from dried blood specimens compared with lymphocytes-possibility for newborn screening. J Inherit Metab Dis 2010;33:43-50.

11. National Committee for Clinical Laboratory Standards. How to define and determine reference intervals in the clinical laboratory: approved guideline, 2001. NCCLS document C28-A and C28-A2, Villanova, PA, NCCLS. 
12. Sözmen EY. Letter to Editor. Sample blank subtraction outreachs Hemoglobin interferences in flurorometric methods for DBS. Mol Genet Metab 2012;105:530.

13. Nakagawa S, Kumin S, Nitowsky HM. Studies on the activities and properties of lysosomal hydrolases in fractionated populations of human peripheral blood cells. Clin Chim Acta 1980;101:33-44.
14. Daitx WV, Mezzalira J, Pereira M, Goldim DS, Coelho JC. Comparison between alpha-galactosidase A activity in blood samples collected on filter paper, leukocytes and plasma. Clin Biochem 2012;45:1233-8.

15. Kleinert J, Ois Dehout F, Schwarting A, et al. Anemia is a new complication in Fabry disease: Data from the Fabry Outcome Survey. Kidney Int 2005;67:1955-60. 